\title{
Representing Causality and Reasoning about Controllability of Multi-level Flow- Systems
}

\author{
Heussen, Kai; Lind, Morten
}

Published in:

2010 IEEE International Conference on Systems, Man, and Cybernetics

Link to article, DOI:

10.1109/ICSMC.2010.5642272

Publication date:

2010

Document Version

Early version, also known as pre-print

Link back to DTU Orbit

Citation $(A P A)$ :

Heussen, K., \& Lind, M. (2010). Representing Causality and Reasoning about Controllability of Multi-level FlowSystems. In 2010 IEEE International Conference on Systems, Man, and Cybernetics: Intelligent Systems for a Safe and Secure World IEEE. https://doi.org/10.1109/ICSMC.2010.5642272

\section{General rights}

Copyright and moral rights for the publications made accessible in the public portal are retained by the authors and/or other copyright owners and it is a condition of accessing publications that users recognise and abide by the legal requirements associated with these rights.

- Users may download and print one copy of any publication from the public portal for the purpose of private study or research.

- You may not further distribute the material or use it for any profit-making activity or commercial gain

- You may freely distribute the URL identifying the publication in the public portal 


\title{
Representing Causality and Reasoning about Controllability of Multi-level Flow-Systems
}

\author{
Kai Heussen and Morten Lind \\ Department of Electrical Engineering \\ Technical University of Denmark \\ Kgs. Lyngby, Denmark \\ $\{$ kh, mli\}@elektro.dtu.dk
}

\begin{abstract}
Safe operation of complex processes requires that operators maintain situational-awareness even in highly automated environments. Automatic reasoning can support operators as well as the automation system itself to react effectively and appropriately to disturbances. However, knowledge-based reasoning about control situations remains a challenge due to the entanglement of process and control systems that co-establish the intended causal structure of a process.

Due to this entanglement, reasoning about such systems depends on a coherent representation of control and process. This paper explains modeling of controlled processes with multilevelflow models and proposes a new framework for modeling causal influence in multilevel flow models on the basis of a flow/potential analogy. The results are illustrated on examples from the domain of electric power systems.

Index Terms-Knowledge-based Systems, Means-ends, Power Systems, Intelligent Control, Model-based reasoning, Causality, Functional Modeling
\end{abstract}

\section{INTRODUCTION}

Security and safety of technological infrastructures and complex processes requires a thorough understanding of their basic physical principles - and just as much of the control systems enabling their operation.

The interconnection of electric power networks over whole continents creates a complex interdependent setting where incidents in one location may have an effect across continent. The control architecture of interconnected power systems therefore counteracts the propagation of disturbances, for example by activating the resources inside the grid region from which the disturbance originated. The electromechanical process interconnects alternating-current synchronous machines in a large area, but a control architecture established on top of that process modifies the causal structure, thereby reducing dangerous interdependence.

This example illustrates the entanglement between process and control systems with respect to causal explanations. Whereas the connection between causality and control is obvious to control engineers, it is only implicit in typical representations used in design documents. For example, signal diagrams which are common in control engineering are based on a strict input-output notion of causality, but their relation to process diagrams is not explicit and requires insight into the mathematical modeling of the specific controlled process.

Thinking in terms of causality is a basis for human understanding of processes. However, the specific understanding of how things are causal in a given domain cannot be generalized to other domains. This is one reason why explicit representation of causality becomes important when multi-disciplinary and multi-domain systems are employed.

Many developments in electric power systems, particularly the move toward more uncontrolled renewable energy sources and the so-called smart grid, tend toward a deeper integration of different domains of energy [1]-[3], where the overall system efficiency and reliability can be improved. New control approaches and changing control architecture are expected [4]; a much wider range of active devices will require a reformulation of current operation principles, which are based on very limited numbers and kinds of devices, to a more functional description of requirements [5]-[10].

These developments also imply new demands on the manageability and controllability of the overall system. Our ability to study, determine and oversee the behavior of a system depends on our ability to represent and thus to model the system's relevant features. As intelligent control is concerned with the control and supervision of systems, including systems that control other systems, it becomes vital to clearly identify the context of representation (the system-in-view).

Knowledge-based systems have a strength in representing human knowledge and thus also to represent information in context. A central challenge of knowledge-based systems application for critical infrastructures is the the lack consistent representation of processes and their control.

\section{A. Qualitative Representation of Processes, Causality and Control}

In this paper we will present a modeling and reasoning approach based on a qualitative model of both process and control systems in a common modeling framework: Multilevel-FlowModeling. Multilevel Flow Modeling (MFM) is a processoriented ontology capturing qualitative functions of material and energy flow processes as well as control functions.

Qualitative representation of processes has some history connected with earlier developments in artificial intelligence. Qualitative Process Theory (QPT) [11] introduced commonsense physics to the description of physical processes. QPT also marked the departure from device-oriented modeling to a process-based abstraction to capture more generic functions of technical processes. 


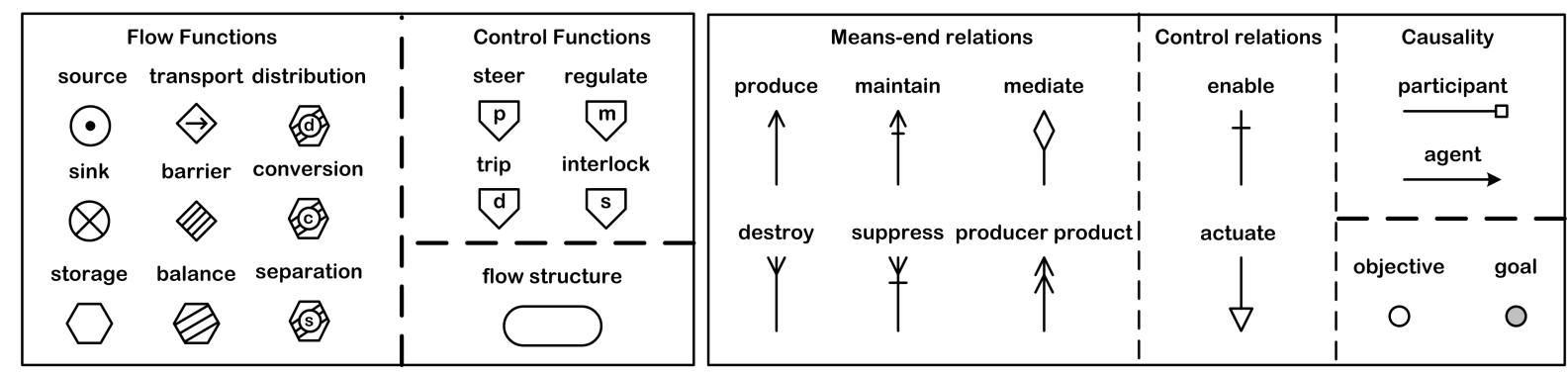

Fig. 1. MFM Entities and Relations.

Representation of material- and energy-flow processes in MFM can be compared to a domain-specific ontology in QPT. The real representational power of MFM, however, comes from its framework of explicit means-ends and partwhole abstractions: Every connected energy- or material-flow is encapsulated in a so-called flow-structure, which then is related with a purpose (an objective, or its function with respect to another flow-structure). These part-whole and meansends patterns describe the two basic abstractions enabled by MFM. Using basic flow-functions and transformation-rules, a process can always be described in further detail; and using a means-end abstractions, a process-hierarchy, such as a controlhierarchy can be captured.

An explicit representation of causal influence within flowstructures has been considered in MFM since [12] and [13], [14]. In [13] the understanding of causal influence is related to QPT and the need for and practicality of generic causation rules are emphasized.

In this paper, a more rigorous formal basis for modeling and reasoning with MFM is proposed and new agent-roles are introduced reflecting the role of a control agents in the process.

Section II introduces Multilevel Flow Modeling and the relevant background concepts. The results of this work are presented in Section III, introducing temptative roles and the concept for causal influence and reasoning. The method is illustrated on two examples, a power plant and a power grid in Section IV.

\section{Multilevel Flow Modeling}

MFM is a functional modeling methodology that provides a library of control functions, energy- or mass-flow-functions and relations, depicted in Figure 1, that can be interconnected to a multi-level representation of causality and intention in flow systems [15]. Adding to the former variety of applications in process engineering, nuclear power plants and others, the field power systems has been developed recently [9], [10]. An MFM model enables situation-dependent reasoning about control situations, by relating system states to system and control objectives.

Applications of MFM include model based situation assessment and decision support for control room operators, hazop analysis [16], alarm design, alarm filtering [17] and planning of control actions [18], [19]. It has been used for knowledge

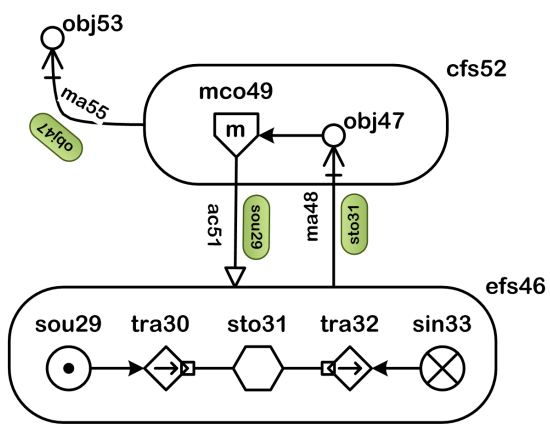

Fig. 2. Example MFM Model with energy flowstructure and control structure. The energy-flowstructure efs 46 models a stereotypical balancing process, where both the energy-source on the left and the energy sink on the right influence the storage-level. In this example, the process is balanced by means of a control which aims at maintaining the storage-level by means of actuating the energy source.

representation in AI planning for supervisory control systems [20].

Altogether MFM provides a rich ontology for modeling purpose-aspects of complex processes. MFM is supported by knowledge based tools for model building and reasoning: a graphical modeling environment and a rule-based reasoning environment with graphical user interface (referred to as MFM Workbench in the following).

\section{A. Modeling of Control in MFM}

A representation of control systems based on action theory has been added more recently to MFM [15], [21], [22]. The four elementary control functions, which are based on elementary action types, are found in Figure 1.

In contrast to the classical signals and systems perspective, control functions have a special role in the perspective of mean-ends modeling: Whereas a 'flow-structure' is a functional abstraction of a process, the 'control-structure' is a representation of the intentional structure realized by a control system $^{1}$. This distinction becomes essential when reasoning about control systems.

An example model of a control structure and a related flowstructure is given in Figure 2:

\footnotetext{
${ }^{1}$ In the control literature, the 'intentional system' is sometimes referred to as 'active' structure, whereas the the controlled system, here '(multi-level) flow-structure', is referred to as the 'passive' basis. This wording does not apply exactly for multilevel-flow-structures, as energy sources and sinks may well be part of the system.
} 


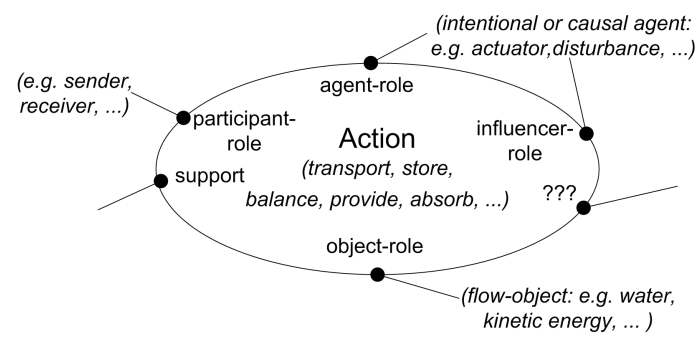

Fig. 3. Action-roles define the participants of an action. Depending on the function and context the roles considered necessary vary.

- Control-objective obj47 and control function mco49 are encapsulated in a control structure cfs52.

- Requirements to the performance of the control are formulated as an objective associated with the control structure (performance objective, obj53).

- The control objective is associated via a means-objectiverelation with the mainfunction (here sto31), the state of the mainfunction is subject of control.

- The control function is connected to the flow-structure via an actuation-relation, ac51, targeting sou29.

In [9], [10] the authors have shown how this modeling of control can be applied to power systems.

\section{B. Functional Roles}

In [14], the connection between the symbolic representations of functions and the semantics of actions have been elaborated:

Definition 1 (Function). A function of an entity $E$ which is part of a system $S$, is specified in terms of the role $R$ of $E$ in relation to an action describing and intended state-change in $S$.

Functions model interconnected actions or actionprimitives. The actions can be associated with a "semantic deep structure" [14], defining roles of an action as slots that can be filled, which is illustrated in Figure 3.

This understanding of a function as an action with a semantic deep structure implies that a number of roles can be associated with each function, such as agent- and object-roles. Further, the action-metaphor is deeper than the flow-metaphor, and potentially enables extension of MFM to other domains of representation if necessary.

Flow-structures are an interconnection of actions with a common flow-object.

Definition 2. (Flow perspective [14]) The flow perspective on an action describes the state change that the object is undergoing without reference to the agent involved.

Flow-functions are formulated in the flow perspective of the actions modeled. A relation between two function-structures therefore also marks a perspective-shift, in which for example the flow-object of another structure turns into an external agent of the related function [3], [23]. As will be shown later, external agents influence the causal structure of a process, and such agents can also be attributed to flow-functions on the basis of control-considerations.

\section{Causality in Flow-models and Causal Reasoning}

Fundamental to the understanding of causality in MFM flow-structures is the notion of agency. Causal roles, as introduced by [14], express the influence that a state of a flow-function has on the flow associated with an adjacent transport function. The role is always marked at the transportside of a connection-line between two functions, ending with a box (participant-role) or with an arrow (agent-role) (shown in Figure 1, on the right: Causality).

A flow-perspective enables causal reasoning over flowsystems, in order to predict consequences or to find possible root-causes of a state-change in the system. This concept of fault diagnosis with MFM was presented in [12], and extended with explicit causal agency in [13], [14]. MFM-based rootcause analysis has been applied for diagnosis and used in commercial applications for alarm filtering.

In past implementations, the causal propagation logic considered interactions between function-pairs, but did not include the role of control agents. In the following, the causal roles introduced in [13] will be utilized, but the logic of influence will be condensed to more rigorous syntactic rules.

\section{Representing Causality and Control}

Even though the larger part of this paper will focus on reasoning about causality within flow-structures, it is important to emphasize the larger perspective that modeling with MFM provides, especially for the modeling of controlled processes.

MFM facilitates the definition of the roles a control system may take with respect to a process (more in Section III-A), as well as the different types of requirements that need to be formulated for a process.

The development of the causal reasoning framework is based on an extension of MFM introducing flexible agentroles in Section III-B. The main result of this work, causal pattern classification and causal path reasoning, is presented in Section III-C. Finally, Sections III-D and III-E present the implemented algorithm and a link to controllability.

\section{A. Control as Disturbance Encapsulation}

In a means-ends framework, control structures can be understood as fact-producers, that is, they transform a goal (intention $Z$ ) into an observable fact (result $Z$ ), see Figure 4. In closed loop control, the control system is supplied with information about deviations from the objective, which enables the rejection of influences contrary to the control objective. In an agent-perspective, a successful control agent has the ability to 'overpower' this disturbance agent (successful encapsulation).

Control design anticipates disturbances and equips the controller with sufficient control resources to defeat expected 


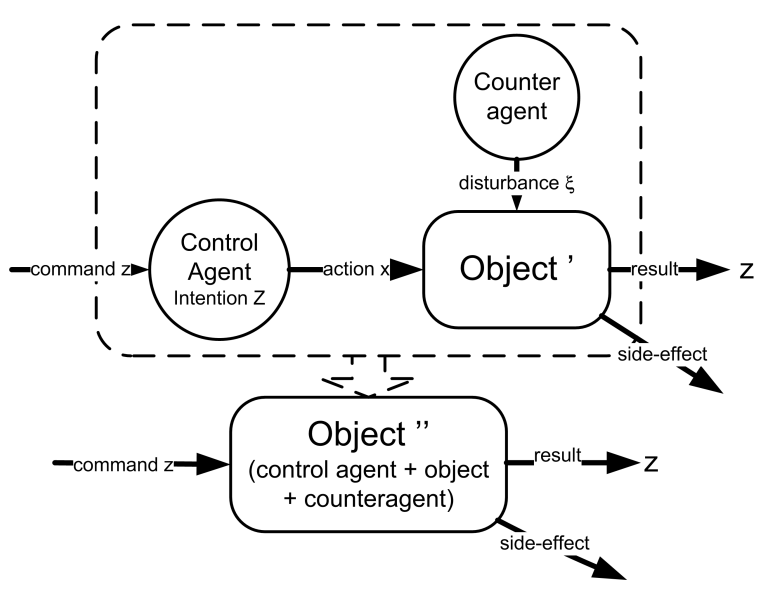

Fig. 4. Encapsulation of disturbance by a control agent. The introduction of a control agent implicitly models a virtual counter-agent.

disturbances. Figure 4 illustrates this concept of control as disturbance encapsulation ${ }^{2}$.

Complex processes are usually composed of several levels of such encapsulation. A higher-level system acts on an encapsulated system, without a need to consider the disturbance that has been encapsulated. This leads to the notion of execution levels. A typical example is a cascaded control system, where the lower-execution level receives an input signal as control reference, and a higher level systems perceives the closed loop of the lower level systems again as dynamical input-output structure. Depending on the level of abstraction, subordinated control loops need not be represented explicitly. The modeling of the feedwater pump control in Example 1, Section IV-A, is another example for this situation.

Reasoning about control levels thus requires a representation of this encapsulation. A necessary condition for this reasoning is thus to frame the causality at the right level of abstraction. For the remainder of this paper we focus on the representation of causality that forms one control level.

\section{B. Introduction of External-Agent Roles}

As outlined above, the action-perspective allows a straightforward extension of multilevel-flow-models to attribute external roles. In the context of control, we establish three new roles capable of influencing the state of a function: Actuator, Disturbant and Conservant, as shown in Figure 5 a). Figure 5 b) illustrates the use of these roles in a simple MFM example, analog to Figure 2.

An actuator performs the commands it receives from a control agent (control function). Therefore it needs to be equipped with a reference to the actuation-relation (multiple roles may refer to the same actuation-relation). It can also be parameterized with a control-range, but quantitative aspects will not be considered in this paper.

\footnotetext{
${ }^{2}$ The term "disturbance rejection" of control engineering is equivalent, but supposes a control-perspective. In a process-perspective, successful control actions render the respective disturbance irrelevant.
}

a)

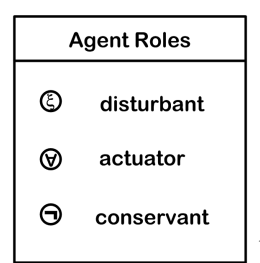

b)

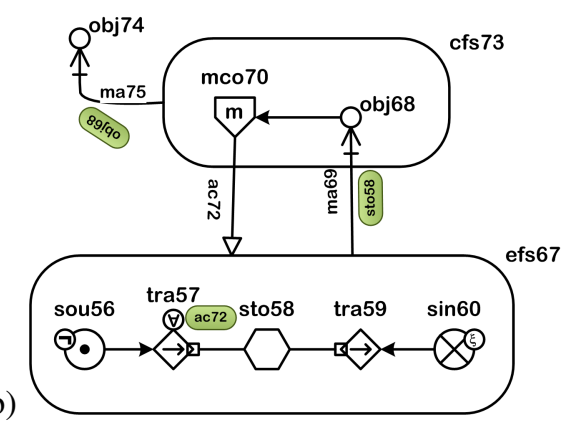

Fig. 5. a) New External-Agent-Roles. b) MFM model, based on Figure 2 with attached roles. Here, the distrubant corresponds to load variations, the conservant corresponds to a setpoint for the source-potential, and the actuator is influencing, not determining, the flow through tra57.

A disturbant represents a disturbance, i.e. the role assumed by the counter-agent. It may also be parameterized with a quantitative information.

The third role-entity, the conservant ensures that the variable, which a control agent would have manipulated through an actuator, is kept static, like a fixed setpoint.

The roles can be attached to these flow-functions (refer to Figure 1 for the complete set): Source, Sink, Transport. Attachment of a role means that a free variable of the respective function is now determined by the external agent who is represented by the role. This also means additional influence on the state-variable associated with a given function is noted, which changes the causal pattern of the function, as seen in the next section.

Storage and Balance do not accept an external role - there are no free variables. The other flow-functions have not been considered yet.

\section{Patterns for Causal Reasoning}

In this section a notion of causality is developed that is suitable for multilevel-flow-models and the modeled processes, but also consistent with underlying physical concepts. It should be noted that, similar to the notions developed in QPT, flowfunctions have been defined from intuitive and generic processengineering notions rather than from physical laws.

The reasoning system classifies patterns within the flowstructures of the MFM model and associates state-variables to the flow-functions.

1) Introduction of State Variables: In order to introduce a logic of influences, we will introduce state-variables to the flow-functions, dependent on the causality pattern surrounding them.

Two types of state variables are introduced: e-/m-flow $(f)$ and potential $(v)$, corresponding to the analogies: mass-flow and mass, as well as energy-flow (power) and energy (content).

The analogy is intuitive, considering an energyflowstructure: We associate an energy-flow with every transport function and a potential with every storage function.

For the remaining functions, the state-variable assignment depends on the surrounding function pattern. 


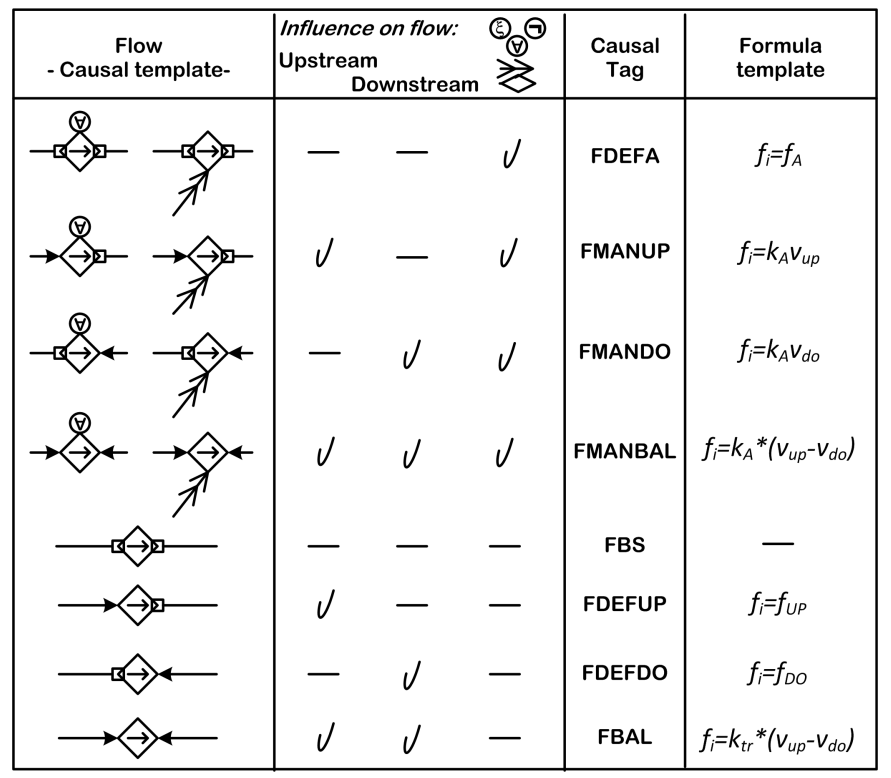

Fig. 6. Classification of causal templates. The templates are differentiated by the origins of influence on their flow: upstream, downstream or external agent.

2) Causal Context of Transport functions: The modes of causation in a MFM-flow-structure are centered around transport-functions, which represent the energy- or mass-flow between any two non-transport functions. The table in Figure 6 lists eight templates that imply a different causal context. Eight, because there are two sides of a transport (upstream or downstream) with two possible roles each (participant or agent), and in addition, there may or may not be an external agent associated with the function. External agents can be roles, as introduced in the previous section, or a meansfunction relation: producer-product or mediate.

The logic behind this classification is apparent: If only one agent is present, it defines the flow in the transport function (FDEFA, FDEFUP, FDEFD). If there are two causal agents, the flow has to be established from a difference in the potentials of the connected flow-functions (FBAL); in addition, the rate of this flow-exchange can be manipulated by an external agent (FMANBAL). The third and last case is derived from the FMANBAL case, but it requires the definition of a neutral potential (FMANUP, FMANDO). Finally, patterns with the the causal tag FBS cannot be accepted for causal reasoning, because there is no causality assigned.

All cases can be illustrated on the examples of connected water-tanks. FDEFA: A pump between two tanks is moving water from one tank to another. FDEFUP, FDEFDO: A watersource, or sink, possibly driven by a pump that would be external to the system-in-view. FBAL, FMANBAL: the classical interconnected tanks, possibly with a valve in the connecting tube. An example of the last case (FMANUP,FMANDO) would be water that is flowing from an outlet at the bottom of a tank - the flow-rate can be manipulated by a valve, but it is also dependent on the water-level.

To present a mathematical analogy of these causal influence

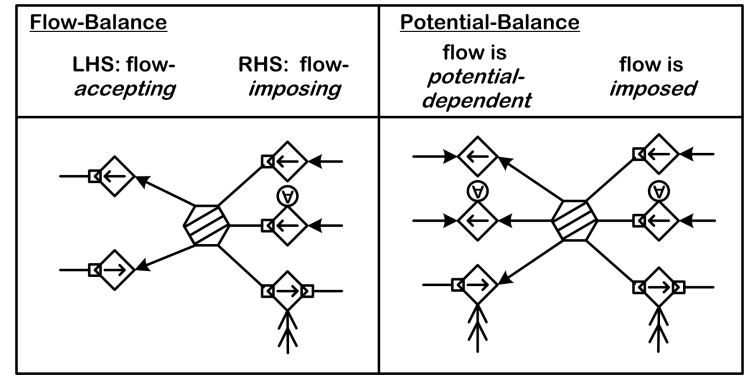

Fig. 7. There a two possible cases for a balance: Flow-balance and Potentialbalance. For each type of influence there are example-transport-functions, for the acceptable patterns (causal tags).

situations, we associate flow-variables $f, v$ for potentials and $k$ to indicate a rate-parameter. The equations on the rightmost column of Figure 6 indicate the analogy. Note that the state of the transport, $f_{i}$, is always the result of causation. It can either be imposed directly, or result from a potential difference of adjacent functions, moderated by a rate-factor. The potentialrate model corresponds to a constitutive equation (such as Ohm's Law).

Based on these templates, state-variables can be assigned to neighboring adjacent sources and sinks: For a FDEFUP(FDEFDO-) transport, an adjacent source (sink) is assigned a flow-state, in all other cases a potential.

3) Flow- and Potential-Balances: For Balance functions, the causal context is analyzed and two types of balances are identified:

- Flow-balance: Pre-assigned causality. A number of flows is imposed (RHS) and are summed up, which defines the flow through the balance: noted in the intermediate flowvariable $f_{b a l}^{*}$.

- Potential-balance: Partly a-causal. Flow is a result of potential differences across the balance and the respective transports. The Balance is assigned an intermediate potential $v_{b a l}^{*}$, analog to hydrodynamic pressure.

The patterns that establish either kind of balance are illustrated in Figure 7. For the flow-balance, a minimum of one connected RHS transport, a transport for which the balance is only participant, is required and the LHS requires exactly one FDEFUP/FDEFDO transport if the flow-direction is always the same, or a second transport with opposing flow-direction. The potential-balance has the same requirement for the RHS (with defined causality), but has a no directly resulting flow.

In case of the flow-balance, the causality structure is that of input-output: a flow-input (RHS: right-hand-side) defines flow-output (LHS: left-hand-side). This can be formulated as

$$
f_{t r, L H S}:=f_{b a l}^{*}:=\sum f_{t r, I N, R H S}-\sum f_{t r, O U T, R H S},
$$

where $f_{i}$ refers to the flow-variable associated with the respective function $i$ of the LHS- or RHS-category of this balance. The resulting flow $f_{b a l}^{*}$ is imposed on the LHS transport(s), depending on directionality. This may be formulated as fol- 
lows:

$$
f_{t r, O U T, L H S}=\left\lfloor f_{t r, L H S}\right\rfloor_{0} \quad \text { and } \quad f_{t r, I N, L H S}=\left\lceil f_{t r, L H S}\right\rceil^{0}
$$

Practically speaking, flow-networks are common where a) system design ensures that no state-feedback happens, i.e. the system is flow-controlled, or potential-differences are too large for variations to matter, and b) there is no choice between potentially alternative flow-recipients/senders (singleoutput requirement).

A potential-balance is a-causal for a part of the connected flows. The flow through the balance is a result of the total potential difference across the balance, so the intermediatepotential at the balance $v_{b a l}^{*}$ is required to determine the flow. In addition, there may be flows imposed to the balance, analog to the RHS of a Flow-balance.

In case of a linear analogue, the intermediate potential $v_{b a l}^{*}$ for Potential-balance would be established as follows:

$$
\begin{aligned}
&\left(\sum k_{T r, U P, i}-\sum k_{T r, D O, i}\right) v_{b a l}^{*}:= \\
&\left(\sum k_{T r, U P, i} v_{U P, i}-\sum k_{T r, D O, i} v_{D O, i}\right) \\
&+\sum f_{t r, I N, R H S}-\sum f_{t r, O U T, R H S},
\end{aligned}
$$

where $k_{T r, U P / D O, i}$ refers to the rates associated with the respective transport (FBAL,FMANBAL,FMANUP/DO) connected to this balance, $v_{U P / D O, i}$ refers to the neighboring potential connected through transport $i$, and the RHS is analog to the flow-balance above.

A network of potential balances corresponds to a linear vector-equation, similar to the load-flow equation of an $\mathrm{AC}$ electricity-network, with a potential-balance assigned to each bus. For a linearized power-flow equation, the 'intermediate potential' $v_{b a l}^{*}$ would correspond to the bus voltage angle variation $\Delta \theta_{\text {bus }}$ (illustrated in Example 2, Section IV).

\section{Propagation of Influence: Influence-tree and Causal-path}

The causal-reasoning system aims at generating a causal path from assigned external agent roles to the objective, that is, to the function associated with this objective (mainfunction).

A system of production rules has been implemented in the MFM Workbench in the rule-based language Jess. The reasoning process can be divided into the following general steps:

1) Analyze causal patterns in all flow-structures in the MFM model: Causal tagging of transports, assignment of state-variables and pattern-identification for flow/potential balances.

2) Initiate: Which control objective is to be traced? The control objective becomes root of the influence-tree.

3) Generate influence-tree for the selected control-objective. Using propagation-rules based templates and patterns identified previously, a tree-structure is generated which notes all possible influences from the model.

4) Trace causal paths in influence tree.

The result of this analysis is a) an influence-tree that contains a reference to all entities that whose state influences

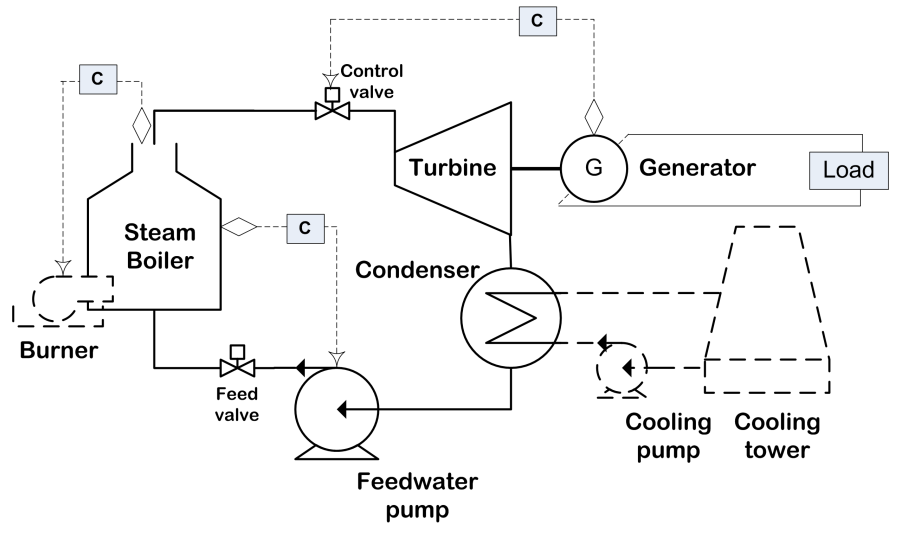

Fig. 8. Process diagram of the thermal power plant in Example 1. For simplicity of illustration, the material flow of dashed components as well as the energy recovery in the feedwater are not modeled here. The objective of the control loop between boiler and feedwater pump is to maintain a constant water-level in the boiler, thus the evaporation rate indirectly determines the water flow in the feedwater pump.

the fulfillment of the objective and $b$ ) a direct path of influence for each external-agent role with influence on the state of the mainfunction (causal path). This reasoning principle will be illustrated in Example 1.

\section{E. On Controllability and the Causal Path}

The concept of controllability is fundamental to control engineering, as it formulates a necessary condition for controlling a system. A full analysis of this concept would be beyond the scope of this paper, but we may reflect on the properties of flow-structures.

A number of different controllability properties are known, including:

State controllability: A dynamical system is called completely controllable if an external input can move the state of the system from any initial state to any other final state in finite time.

Output-controllability: analog to state-controllability, but instead of the full systems state, the system's output is required to be moved.

We do understand inputs as assigned actuator-roles and outputs as the states of the mainfunction associated with the control-objective. The "system" could be considered exactly those functions that are part of the influence-tree.

Under certain -limiting- conditions, there is a mapping to output-controllability: a) there are no causal loops in the system b) there is only one actuator in the tree.

A detailed study of the graph properties of flow-structures and their mapping to linear systems could generate further structural sufficient conditions for controllability.

\section{EXAMPLES}

The modeling and reasoning principles shall be demonstrated on some examples. The first example highlights the multi-level physical representation aspects of the modeling approach on a simplified power-plant. The second example 


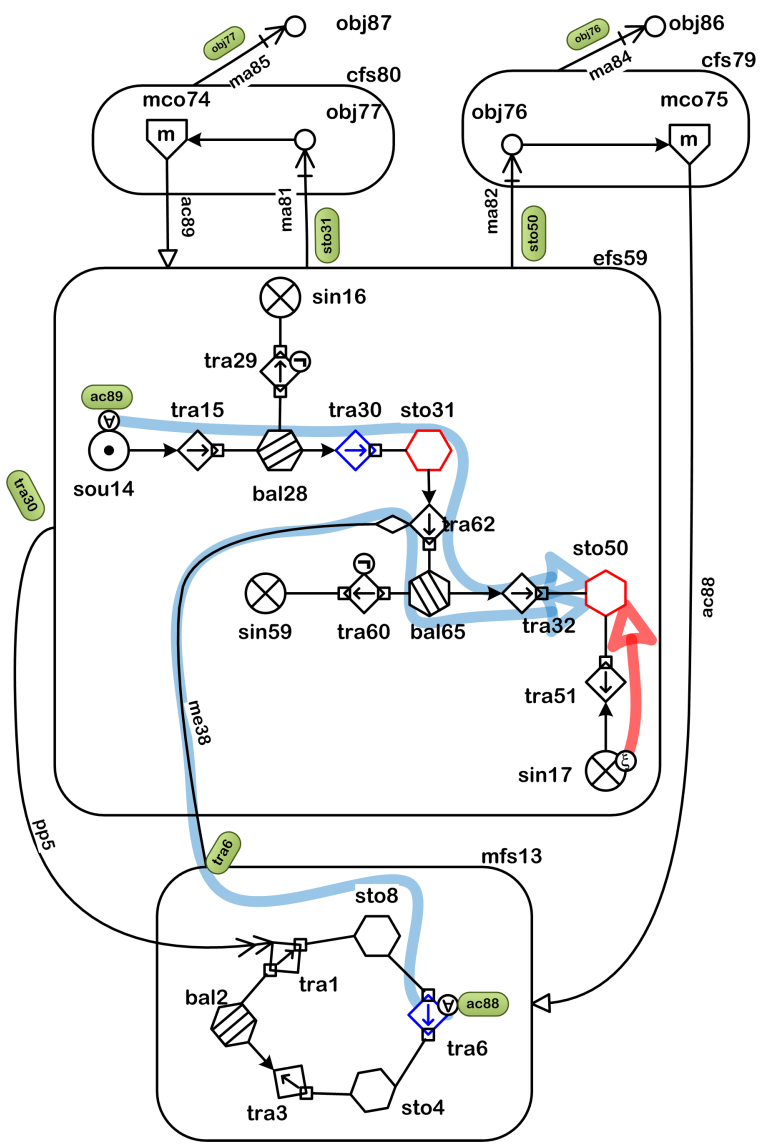

Fig. 9. MFM model for the main control loops of the thermal power plant. Please note that the green bubbles contain a reference to a related entity. The boiler-feedwater control-loop displayed in Figure 8 is not modeled as a control loop but function for the system, illustrating the consideration of abstraction levels introduced in Section III-A. The functions bal2 and tra3 and their causal relations, capture the effect that always as much water is pumped into the boiler as is being evaporated. The wide arrows in the background illustrate the causal paths for obj76 from the actuator- and disturbant-agents.

illustrates the opportunities of modeling mixed causality structures (potential and flow) of networks, such as electric energy systems with both DC and AC links.

\section{A. Example 1: Power Plant}

In this example we model the main control loops of a thermal power plant supplying a varying electrical load in island mode ${ }^{3}$. The modeled process is illustrated in Figure 8. The power plant model is simplified by assuming a fixed cooling- and smoke-power loss.

An MFM-model of the process is presented in Figure 9. The model comprises two flow-structures, modeling the process at the relevant abstraction level, and two control structures representing the main control loop objectives of the power plant: fresh-steam pressure setpoint and frequency control (to encapsulate the "disturbance" of varying power demand).

1) Model Description: The lower flow-structure, mfs13, models the mass-flow of the main water-circulation. There are

\footnotetext{
${ }^{3}$ This leads to direct "isochronous" frequency control, otherwise, the controller would adjust the power-output of the plant, see also [9], [10]
}

two mass-storages in the loop: sto8 and sto4, representing fresh steam mass and cold-steam/condensate, respectively. Balance function bal2 represents the balance between evaporation tra1, driven (pp5) by the heat transfer tra30, and feedwater flow (tra3), which is enabled by the underlying feedwater pump control. The controlled turbine inlet valve is actuated by ac88 to determine the mass-flow of steam to the turbine (tra6). This mass-flow mediates the inflow of energy to the turbine (tra62), as represented by the mediate-relation me38.

The energy flow-structure efs59 models heat-inflow from the combustion of fuel (sou14), heat-loss into exhaust gas (tra29, sin16), heat-transfer to water (tra30) and the steam enthalpy in sto31. The energy-transport to the turbine is influenced by sto31 and me38 (causal-tag: FMANUP, see Fig 6), mass-flow and energy-content. Assuming a static energyloss (tra60, sin59), a fraction of the energy-flow is transferred by the turbine (tra32) to the inertia of the rotating turbinegenerator (sto50), which receives and provides energy to the load (tra51, sin17) without influence from the rotation speed.

Five external agent-roles are attached to functions in this model: Actuators on sou14determining the energy-flow, and on tra6 determining its mass-flow; a disturbant determining the energy-flow into sin17, representing the load variation; conservants determining the energy-flow through tra29 and tra60, representing the assumption of fixed energy-losses.

2) Controllability Analysis: The causal-path analysis (see also arrows in Figure 9) reveals that load-variation, the disturbant on $\sin 17$ does influence the state of sto50 (kinetic energy, frequency), but not the state of sto31 (steam pressure). The intended behavior of the power plant, is to adjust its energy conversion setpoint (eventually, the fuelsupply, sou14) according to the load-demand, a demand-driven process which requires upstream-propagation of information. This upstream-propagation is provided by the two inter-leaved control-structures with control -objectives obj76 and obj77 aiming to determine the energy-states of sto50 and sto31. By manipulation of the mass-flow of tra6 and the supplied energyflow of sou14 the control functions effect the process on its upstream end.

\section{B. Example 2: Power network}

This example of an AC power network connected with an HVDC-link to another AC system (Figure 10), is presented to illustrate the value of a modeling tool that can model systems in terms of their causal interconnection. The flow across the HVDC line power can be controlled and may thus play a role in the overall control architecture.

It also shows how the flow-structure representation is analog to a "DC-power-flow", a linearized power flow formulation. Here, power exchange is solely driven by voltage-angles, corresponding to the potential-variables introduced above.

\section{CONCLUSION}

This paper presented an extension of the causality concept of Multilevel-Flow-Models and the according reasoning tool for the purpose of controllability analysis. The underlying 


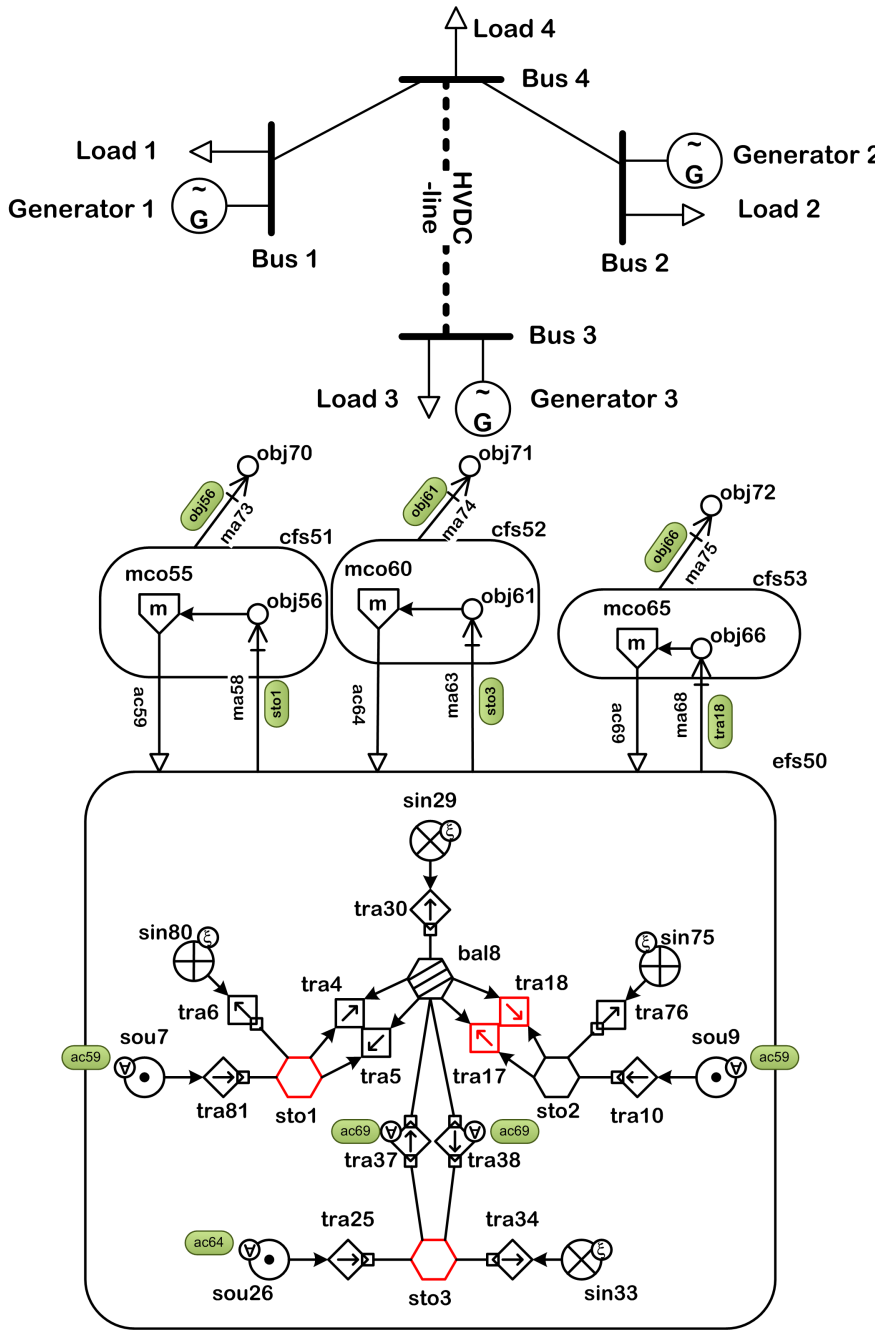

Fig. 10. One line diagram and MFM model, illustrating the network modeled in Example 2. Note the combination of two AC networks (synchronous areas) with a DC link. In the MFM model, mainfunctions associated with the control objectives are marked in red.

conceptual model introduces flow- and potential variables to make sense of the influence patterns modeled by the causal roles of an MFM model. Based on this idea, syntactic rules for function-causality configurations have been derived.

The concepts have been implemented in a softwareframework which enables graphical modeling and model-based reasoning for artificial intelligence applications.

The method has been demonstrated on two energy systems examples. It has been shown that the modeling approach readily maps into the domain-specific physical frameworks.

Further we have outlined, how the causal-path concept introduced in this paper is related to the controllability concept of control engineering. A flexible assignment of agent roles allows the re-use of models and restructuring of control-loops and objectives on a given process-model.

\section{ACKNOWLEDGMENT}

The work presented in this paper is a result of the research project Coherent Energy and Environmental System Analysis
(CEESA), partly financed by The Danish Council for Strategic Research.

\section{REFERENCES}

[1] D. Pudjianto, C. Ramsay, and G. Strbac, "Virtual power plant and system integration of distributed energy resources," IET Renew. Power Gener., vol. 1, no. 1, pp. 10-16, 2007.

[2] M. Geidl, "Integrated modeling and optimization of multi-carrier energy systems," Ph.D. dissertation, ETH Zurich, 2007.

[3] K. Heussen and M. Lind, "Functional modeling of perspectives on the example of electric energy systems," in Zero-Carbon Energy Kyoto, T. Yao, Ed. Springer, 2009.

[4] M. D. Ilic, "From hierarchical to open access electric power systems." Proceedings of the IEEE, Special Issue on "Modeling, Identification, and Control of Large-Scale Dynamical Systems", vol. 95, no. 5, pp. 10601084, May 2007.

[5] O. Gehrke, "Infrastructures for power system integration and control of small distributed energy resources," Ph.D. dissertation, Technical University of Denmark, 2009.

[6] I. Kamphuis, J. Kok, C. Warmer, and M. Hommelberg, "Architectures for novel energy infrastructures: Multi-agent based coordination patterns," in IEEE-NGI, Rotterdam, The Netherlands. ECN, 10-12 November 2008, presented, paper available at http://www.ecn.nl/publications/.

[7] R. Gustavsson, "Ensuring quality of service in service oriented critical infrastructures," in The International Workshop on Complex Network and Infrastucture Protection (CNIPt'O6), 2006.

[8] M. Lind, T. Ackermann, P. Bach, H. W. Bindner, Y. Chen, R. GarciaValle, M. Gordon, K. Heussen, P. Nyeng, A. Saleem, P. E. Sørensen, M. Togeby, I. Vlachogiannis, S. You, and Z. Xu, "Ecogrid.dk phase I WP2 report - system architecture," Energinet.dk, Tech. Rep., 2008.

[9] K. Heussen, A. Saleem, and M. Lind, "Control architecture of power systems: Modeling of purpose and function," in Proceedings of the IEEE PES General Meeting 2009, 2009.

[10] K. Heussen and M. Lind, "Decomposing objectives and functions in power system operation and control," in Proceedings of the IEEE PES/IAS Conference on Sustainable Alternative Energy, Valencia, 2009.

[11] K. Forbus, "Qualitative process theory," Artificial Intelligence, vol. 24, pp. 85-168, 1984.

[12] M. Fang and M. Lind, "Model based reasoning using MFM," in Proc. Pacific-Asian Conference On Expert System (PACES), Huangshan, China, 1995.

[13] J. Petersen, "Causal reasoning based on mfm," in Proceedings of Cognitive Systems Engineering in Process Control (CSEPC), 2000, 2000.

[14] _ "Knowledge based support for situation assessment in human supervisory control," Ph.D. dissertation, Department of Automation, Technical University of Denmark, Lyngby, Denmark, 2000.

[15] M. Lind, "A goal-function approach to analysis of control situations," in Proceedings of 11th. IFAC/IFIP/IFPRS/IEA Symposium on Analysis, Design and Evaluation of Human-Machine Systems, 2010.

[16] N. L. Rossing, M.Lind, N. Jensen, and S. B. Jørgensen, "A goal based methodology for hazop analysis," in Proc. 4.th International Symposium on Cognitive System Engineering Approach to Power Plant Control (CSEPC2008), Harbin, Heilongjiang, China, September 8-10 2008.

[17] J. E. Larsson, "Diagnosis based on explicit means-end models." Artificial Intelligence, vol. 80(1), pp. 29-93, 1996.

[18] M. N. Larsen, "Deriving action sequences for start-up using multilevel flow models," Ph.D. dissertation, Department of Automation, Technical University of Denmark, 1993.

[19] A. Gofuku and Y. Tanaka, "Development of an Operator Advisory System: Finding Possible Counter Actions in Anomalous Situations," in Proc. 5'th International Workshop on Functional Modeling of Complex Technical Systems, Paris, France, July 1-3 1997, pp. 87-97.

[20] L. E. de Souza and M. M. Veloso, "AI planning in supervisory control systems," in Proc. IEEE International Conference on Systems, Man and Cybernetics, Beijing, October 14-15 1996, pp. 3153-3158.

[21] M. Lind, "Means and ends of control," in Proc. IEEE Conf. Systems Man and Cybernetics, The Hague, Holland, October 10-13 2004.

[22] — , "Modeling goals and functions of control and safety systems in MFM," in Proceedings International Workshop on Functional Modeling of EngineeringSystems, Kyoto, Japan, January 25 2005, pp. 1-7.

[23] _ "Plant modeling for human supervisory control," Transactions of the Institute of Measurement and Control, vol. 21, no. 4-5, pp. 171-180, 1999. 